\title{
Resultados alejados de la reparación versus reemplazo en insuficiencia mitral degenerativa
}

\author{
Ricardo Zalaquett S, Maximiliano Scheu Ga, \\ Cristóbal Camplá Ca, Sergio Morán V, Manuel J Irarrázaval LI, \\ Pedro Becker R, Claudio Arretz V, Samuel Córdova A, \\ Sandra Braun J, G astón Chamorro S, Iván G odoy J. \\ Long-term results of repair versus \\ replacement for degenerative mitral \\ valve regurgitation
}

Background: Mitral valve repair is considered better than mitral valve replacement for degenerative mitral regurgitation. Aim: To evaluate late clinical results of mitral valve repair as compared to mitral valve replacement in patients with degenerative mitral regurgitation. Patients and methods: All patients subjected to open heart surgery for degenerative mitral regurgitation between 1990 and 2002 were assessed for surgical mortality, late cardiac and overall mortality, reoperation, readmission to hospital, functional capacity and anticoagulant therapy. Eighty eight patients (48 males) had mitral valve repair and 28 (19 males) had mitral valve replacement (23 with a mechanical prosthesis). Mean age was $59.9 \pm 14.8$ (SD) and $61.3 \pm 14.6$ years, respectively. Sixty three percent of patients with repair and $50 \%$ of those with valve replacement were in functional class III or IV before surgery. Results: Operative mortality was $2.3 \%$ for mitral valve repair and $3.6 \%$ for mitral valve replacement (NS). Also, there was no statistical difference in the need of reoperation during the follow-up period between both procedures ( $2.3 \%$ and $0 \%$, respectively). Ninety four percent of the replacement patients but only $26 \%$ of the repair patients were in anticoagulant therapy at the end of the follow-up period ( $p$ $<0.001$ ). Ten years survival rates were $82 \pm 6 \%$ for mitral valve repair and $54 \pm 11 \%$ for replacement. The corresponding cardiac related survival rates were $89 \pm 6 \%$ and $79 \pm 10 \%$. At the end of follow-up, all surviving patients were in functional class I or II. Ten years freedom from cardiac event rates (death, cardiac related rehospitalization and reoperation) were $90 \pm 3 \%$ for mitral valve repair and $84 \pm 6 \%$ for replacement. Conclusion: Repair of the mitral valve offers a better overall survival and a better chance of freedom from cardiac events as well as need for anticoagulation 10 years after surgery (Rev Méd Chile 2005; 133: 1139-46).

(Key Words: Mitral valve; Mitral valve insufficiency; Thoracic surgery)

Recibido el 17 de marzo de 2005. Aceptado el 30 de mayo de 2005.

Departamento de Enfermedades Cardiovasculares, Facultad de Medicina, Pontificia Universidad Católica de Chile. Santiago de Chile.

aAyudante Alumno, Escuela de Medicina.

Correspondencia a: Dr. Ricardo Zalaquett. Marcoleta 367, $6^{\circ}$

piso. Teléfonos: 6333030 - 3543231. Fax: 6390108. 
A partir de los trabajos pioneros de Carpentier, la reparación mitral se impuso progresivamente por sobre el reemplazo protésico de la válvula mitral como tratamiento de la insuficiencia de ésta, por cuanto permite la conservación de la integridad del aparato valvular mitral y preserva la geometría de la cavidad ventricular izquierda, lo que se traduce en una mantención y mejoría de la función del ventrículo izquierdo, además de no requerir anticoagulación de por vida. Esto, a su vez, se ha traducido en mejores resultados clínicos inmediatos y alejados ${ }^{1-4}$. Posteriormente, se observó que el grupo de pacientes en los cuales es más factible efectuar una reparación mitral exitosa y sostenida en el tiempo, son aquellos portadores de una insuficiencia mitral degenerativa mixomatosa, que en la actualidad ha pasado a ser la principal etiología de la insuficiencia mitral ${ }^{4-6}$.

En 1994, publicamos nuestros resultados inmediatos con la cirugía reparadora de la insuficiencia de la válvula mitral, experiencia iniciada en 1991, poniendo énfasis en los aspectos quirúrgicos ${ }^{7}$. En 1996 publicamos los resultados precoces, dando importancia particular a los aspectos ecocardiográficos preoperatorios ${ }^{8}$. En 1999, comunicamos nuestros resultados tardíos, incluyendo todos los pacientes en los que se efectuó una reparación mitral, con especial atención en su sobrevida y resultados ecocardiográficos alejados ${ }^{9}$. Recientemente publicamos los resultados clínicos y ecocardiográficos alejados de la cirugía reparadora en pacientes con insuficiencia mitral mixomatosa exclusiva ${ }^{10}$.

Presentamos ahora los resultados clínicos tardíos de la reparación valvular mitral en comparación al reemplazo protésico de la válvula mitral, para pacientes con insuficiencia mitral degenerativa, en un estudio retrospectivo, observacional y descriptivo. Dada las características del trabajo y el período de tiempo que comprende, no se incluyeron parámetros ecocardiográficos auriculares ni ventriculares.

\section{Pacientes y MÉTOdo}

Pacientes. Todos los pacientes operados en nuestra institución con insuficiencia mitral degenerativa mixomatosa entre enero de 1990 y diciembre de 2002, fueron evaluados en cuanto a mortalidad operatoria (intrahospitalaria o hasta 30 días de la operación), mortalidad alejada total y cardíaca, necesidad de reoperación cardiovascular, rehospitalización por causa cardiológica, capacidad funcional (CF) al término del seguimiento $\mathrm{y}$ necesidad de tratamiento anticoagulante crónico.

Se identificaron 116 pacientes intervenidos por insuficiencia mitral mixomatosa, los que representaron el $22 \%$ del total de los procedimientos mitrales por insuficiencia mitral del peńodo. Ochenta y ocho pacientes $(76 \%)$ tuvieron una reparación de su insuficiencia mitral y 28 (24\%) un recambio valvular protésico. La decisión de efectuar una reparación 0 un reemplazo mitral fue a criterio del cirujano, dependiendo de factores clínicos y anatómicos, así como de su entrenamiento en reparaciones valvulares. Por esto, la mayonía de los reemplazos mitrales se concentran en los primeros años del estudio.

La edad promedio de los pacientes con reparación mitral fue 59,9 \pm 14,8 años (margen: 22 a 82 años) y la de los con reemplazo mitral de $61,3 \pm$ 14,6 años (margen: 17 a 85 años). El 63\% de los pacientes con reparación y $50 \%$ de los con reemplazo mitral protésico se encontraban en $\mathrm{CF}$ III-IV previo a la operación.

Método. Los pacientes con reemplazo mitral fueron identificados a través de la base de datos general del Servicio de Cirugía Cardiovascular del Hospital Clínico de la Pontificia Universidad Católica de Chile. Por su parte, los pacientes con reparación mitral por insuficiencia mitral degenerativa, fueron identificados de la base de datos específica de reparación mitral del mismo Servicio y representaron $53 \%$ del total de las reparaciones mitrales del período. Identificados los pacientes, se revisaron las fichas clínicas y protocolos operatorios.

Para el seguimiento clínico, los pacientes, sus familiares o médico tratante fueron contactados personal o telefónicamente entre marzo y junio de 2003, solicitándoseles responder un cuestionario de evaluación clínica. Finalmente, se solicitó información al Registro Civil e Identificación de Chile respecto a mortalidad.

El seguimiento se completó en $100 \%$ de los casos con un promedio de 53,6 $\pm 32,6$ meses (mediana: 41,9 meses; margen: 2,5 a 128 meses) para la reparación mitral y de $80,7 \pm 45,7$ meses, con una mediana de 83,6 meses y un margen de 9,3 a 162,2 meses, para el reemplazo mitral. 
Técnica quirúrgica. Todos los pacientes fueron operados con circulación extracorpórea, con hipotermia sistémica entre 28 y $30^{\circ} \mathrm{C}$. La protección miocárdica se efectuó con solución cardiopléjica cristaloide infundida a $4^{\circ} \mathrm{C}$ por vía anterógrada y también retrógrada, en casi todos los casos ${ }^{11}$. En la mayoná de los casos el acceso a la aurícula izquierda fue a través de una auriculotomía transeptal y superior combinada ${ }^{12}$. La reparación de la válvula mitral se efectuó de acuerdo a los principios y técnicas establecidas por Carpentier, con control ecocardiográfico transesofágico intraoperatorio en todos los casos.

En 85 casos (97\%) de reparaciones mitrales se utilizó un anillo de Carpentier Edwards. En 73 de éstos se utilizó el modelo Classic y en 12 el nuevo modelo flexible Physio-Ring. De los 28 pacientes con reemplazo mitral, en 23 (82\%) se utilizó una prótesis mecánica y en los otros 5 una prótesis biológica de Carpentier-Edwards.

Finalmente, en 21 pacientes (24\%) con reparación mitral y en 9 (32\%) con recambio mitral se efectuó una revascularización miocárdica asociada $(\mathrm{p}=\mathrm{NS})$.

La Tabla 1 resume las principales características demográficas, clínicas y quirúrgicas de los pacientes.
Estadística. Los datos se expresan como promedio \pm desviación estándar y la sobrevida se analizó mediante el método de Kaplan-Meier. Para las comparaciones de mortalidad operatoria, mortalidad alejada, reoperación, rehospitalización cardiovascular, CF tardía y tratamiento anticoagulante crónico, se utilizó el test no paramétrico de sumas de rangos de Wilcoxon, considerándose significativo un valor $\mathrm{p}<0,05$. Para la presentación de los resultados se siguieron las recomendaciones del comité conjunto de la American Association for Thoracic Surgery y la Society of Thoracic Surgeons ${ }^{13}$.

\section{RESULTADOS}

La Tabla 2 resume la mortalidad operatoria y la mortalidad global, cardíaca y no cardíaca, para la reparación y el reemplazo mitral, para todo el período en estudio.

Mortalidad operatoria. La mortalidad intrahospitalaria o hasta 30 días de la operación fue similar para la reparación mitral, 2,3\% (2 pacientes) que para el reemplazo mitral, 3,6\% (1 paciente).

\section{Tabla 1. C aracterísticas demográficas, clínicas y quirúrgicas de 116 pacientes operados por insuficiencia mitral degenerativa}

\begin{tabular}{|lcccc|}
\hline & \multicolumn{2}{c}{ Reparación } & \multicolumn{2}{c|}{ Reemplazo } \\
\hline Pacientes & \multicolumn{2}{c}{$78 \%$} & $76 \%$ & \multicolumn{2}{c|}{28} & $24 \%$ \\
Edad promedio & \multicolumn{2}{c}{$59,9 \pm 14,8$} & \multicolumn{2}{c|}{$61,3 \pm 14,6$} \\
Hombres & 48 & $55 \%$ & 19 & $68 \%$ \\
CF III-IV & 52 & $63 \%$ & 8 & $50 \%$ \\
Prótesis mecánica & - & - & 23 & $82 \%$ \\
Prótesis biológica & - & - & 5 & $18 \%$ \\
Anillo Carpentier-Edwards Classic & 73 & $83 \%$ & - & - \\
Anillo Carpentier-Edwards Physio-Ring & 12 & $14 \%$ & - & - \\
Revascularización asociada & 21 & $24 \%$ & 9 & $32 \%$ \\
\hline
\end{tabular}

Tabla 2. M ortalidad operatoria y alejada de 116 pacientes operados por insuficiencia mitral degenerativa

\begin{tabular}{|lcrrrc|}
\hline & \multicolumn{2}{c}{ Reparación } & \multicolumn{2}{c|}{ Reemplazo } & $\mathrm{p}$ \\
\hline Mortalidad operatoria & 2 & $2,3 \%$ & 1 & $3,6 \%$ & $\mathrm{NS}$ \\
Mortalidad global & 8 & $9,1 \%$ & 12 & $43 \%$ & $<0,05$ \\
*Mortalidad cardíaca & 3 & $3,5 \%$ & 7 & $26 \%$ & $<0,05$ \\
*Mortalidad no cardíaca & 3 & $3,5 \%$ & 4 & $14,8 \%$ & NS \\
\hline
\end{tabular}


Mortalidad global. La mortalidad total, incluyendo la perioperatoria, fue de $9,1 \%$ (8 pacientes) para la reparación y de 43\% (12 pacientes) para el reemplazo mitral, $(p<0,05)$. Así, la probabilidad de estar vivo a 5 años fue de $88 \% \pm 4 \%$ y a 10 años de $82 \% \pm 6 \%$ para la reparación y de $80 \% \pm$ $8 \%$ y $54 \% \pm 11 \%$, respectivamente para el reemplazo mitral (Figura 1).

Mortalidad cardíaca. Tres pacientes $(3,5 \%)$ con reparación mitral y 7 (26\%) con reemplazo de la válvula mitral fallecieron por causa cardiovascular durante el período de seguimiento $(p<0,05)$. La probabilidad de estar libre de muerte cardiovascular fue de $94 \% \pm 3 \%$ y $89 \% \pm 6 \%$ a 5 y 10 años para la reparación mitral y de $92 \% \pm 5,5 \%$ y $79 \% \pm 10 \%$ para el reemplazo, para igual período de seguimiento (Figura 2).

Reoperación. Ningún paciente con reemplazo mitral fue reoperado durante el seguimiento. Por el contrario, 2 pacientes con reparación mitral requirieron ser reintervenidos. Uno de éstos fue reoperado al mes y medio de su reparación mitral, por un desprendimiento del anillo de Carpentier-Edwards y la otra lo fue a los 5 meses, por una endocarditis asentada en el anillo de Carpentier-Edwards. En el primero se efectuó un reemplazo mitral y en la segunda solo una remoción del anillo protésico. Este último caso correspondió al único caso de
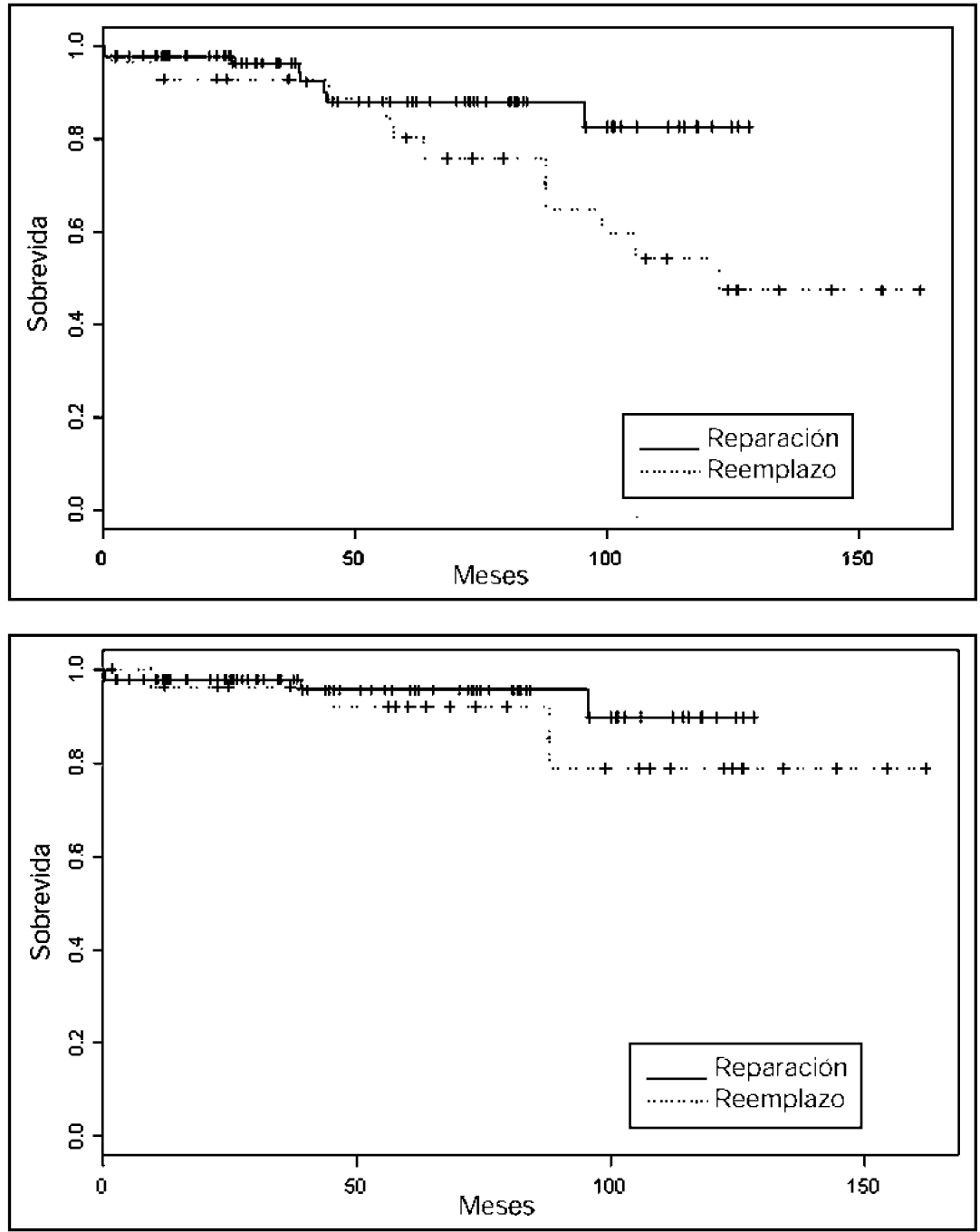

FiguRA 1. Curva de sobrevida actuarial (Kaplan-Meier) global para pacientes con reparación y reemplazo valvular mitral por insuficiencia mitral degenerativa.
Figura 2. Curva de probabilidad actuarial (Kaplan-Meier) de estar libre de muerte de causa cardíaca para pacientes con reparación y reemplazo valvular mitral por insuficiencia mitral degenerativa. 
endocarditis de toda la serie. La probabilidad de estar libre de reoperación para la reparación mitral fue de $98 \% \pm 1,6 \%$ a 1,5 y 10 años.

Rehospitalización. El 34\% de los pacientes con reparación mitral y el 50\% de los con reemplazo fue alguna vez rehospitalizado durante el período de seguimiento. Sin embargo, sólo 3 pacientes $(3,8 \%)$ de los con reparación mitral lo fue por una causa cardiovascular. Por el contrario, 7 pacientes $(43,8 \%)$ de los sobrevivientes con reemplazo mitral fueron rehospitalizados por motivos cardiovasculares ( $p<0,001)$.
La probabilidad de estar libre de rehospitalización por causas cardiovasculares fue de $96 \% \pm$ $2,4 \%$ a 5 y 10 años para la reparación mitral y de $89 \% \pm 6,2 \%$ y $79 \% \pm 11 \%$ para el reemplazo mitral, para iguales intervalos de tiempo (Figura 3).

Eventos cardiovasculares. La probabilidad de estar libre de eventos cardiovasculares, esto es, muerte cardíaca, rehospitalización cardiovascular y reoperación fue de $90 \% \pm 3 \%$ y de $84 \% \pm 6 \%$ para la reparación mitral y de $71 \% \pm 8,6 \%$ y $43 \% \pm 10 \%$ para el reemplazo mitral, a 5 y 10 años, respectivamente (Figura 4).
Figura 3. Curva de probabilidad actuarial (Kaplan-Meier) de estar libre de rehospitalización por causas cardiovasculares para pacientes con reparación y reemplazo valvular mitral por insuficiencia mitral degenerativa.

Figura 4. Curva de probabilidad actuarial (Kaplan-Meier) de estar libre de eventos cardiovasculares para pacientes con reparación y reemplazo valvular mitral por insuficiencia mitral degenerativa.
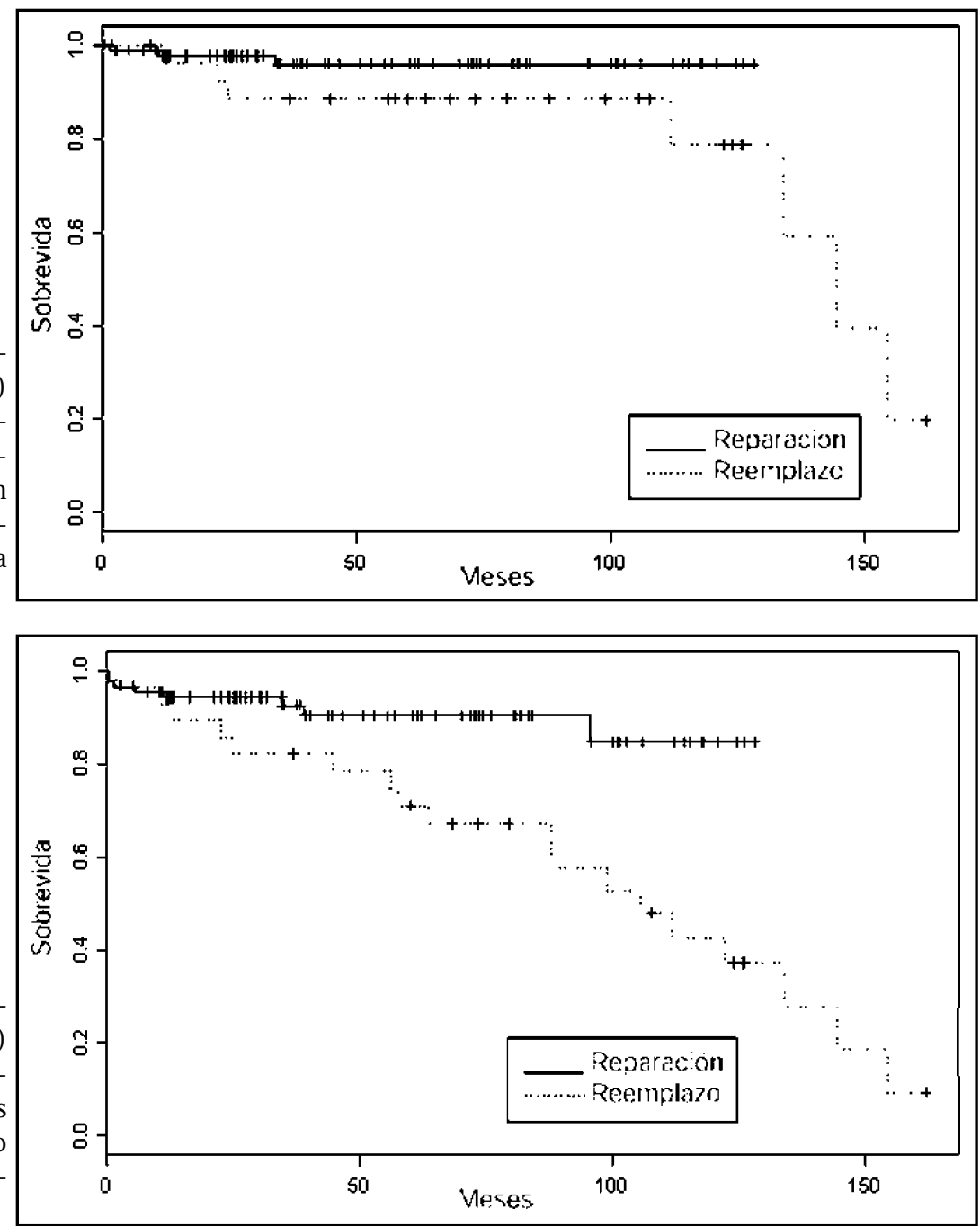
Tabla 3. Reoperación, rehospitalización y tratamiento anticoagulante de pacientes sobrevivientes a operación por insuficiencia mitral degenerativa

\begin{tabular}{|lrrrrc|}
\hline & \multicolumn{2}{c}{ Reparación } & \multicolumn{2}{c|}{ Reemplazo } & $\mathrm{p}$ \\
\hline Reoperación & 2 & $2,3 \%$ & 0 & NS & \\
Rehospitalización total & 27 & $34 \%$ & 8 & $50 \%$ & NS \\
Rehospitalización cardíaca & 3 & $3,8 \%$ & 7 & $43,8 \%$ & $<0,001$ \\
Tratamiento anticoagulante & 21 & $26 \%$ & 15 & $94 \%$ & $<0,001$ \\
\hline
\end{tabular}

Tratamiento anticoagulante y CF. Quince de los 16 pacientes con recambio mitral vivos al término de seguimiento estaban en tratamiento anticoagulante, esto es, $94 \%$ de los pacientes. Por el contrario, sólo 21 de los 80 sobrevivientes (26\%) con reparación mitral estaban en tratamiento anticoagulante crónico al cierre del seguimiento ( $p<0,001$ ). Por otra parte, no hubo diferencia significativa entre los sobrevivientes de ambos procedimientos en cuanto a su $\mathrm{CF}$, encontrándose todos en CF I-II. Previo a la operación, 63\% de los pacientes con reparación y $50 \%$ de los con reemplazo mitral, se encontraban en CF III-IV.

La Tabla 3 resume la incidencia de reoperación, rehospitalización y necesidad de tratamiento anticoagulante crónico durante el período de seguimiento para ambos procedimientos mitrales.

\section{DiscUSIÓN}

La reparación de la válvula mitral es en la actualidad el tratamiento quirúrgico de elección para la insuficiencia de ésta ${ }^{4}$. La cirugía pretende corregir la insuficiencia mitral tratando el mecanismo anátomo-patológico responsable, independientemente de su etiología ${ }^{1-3}$. La ventaja de la reparación por sobre el reemplazo mitral estaría dada por la conservación de toda la estructura anatómica del aparato mitral, el que no sólo permite un flujo unidireccional de sangre desde la aurícula al ventrículo izquierdo, sino que, además, participa importantemente en la eficiencia de la función de bomba de éste, lo que se traduciría en una menor morbimortalidad operatoria y alejada ${ }^{2-6}$.

La degeneración mixomatosa de la válvula mitral es actualmente la principal causa de la insuficiencia de ésta y su incidencia probablemente aumente con el envejecimiento progresivo de la población, si bien no es aún el caso en nuestra institución ${ }^{4-6}$. Por otra parte, es en este grupo de pacientes en los que es más importante preservar la función del ventrículo izquierdo puesto que a diferencia de la insuficiencia mitral isquémica, la disfunción ventricular no es la causa de la insuficiencia mitral, por lo que la mantención o no de la función ventricular izquierda marcará fuertemente la expectativa de vida de cualquier paciente ${ }^{4-6,14}$. Además, es en la insuficiencia mitral degenerativa en la que los procedimientos reconstructores diseñados por Carpentier alcanzan su máxima aplicabilidad, acompañándose de una alta probabilidad de éxito precoz, el que se mantiene a lo largo del tiempo ${ }^{1-6,10}$.

De aquí que, habiendo recientemente analizado y comunicado nuestros resultados alejados de la cirugía reparadora de la insuficiencia mitral degenerativa, quisiéramos en esta oportunidad presentar estos mismos resultados en comparación ahora al reemplazo mitral protésico ${ }^{10}$.

Pacientes. Si bien hubo una importante diferencia en el número total de pacientes con reparación y reemplazo, éstos fueron muy similares en cuanto a edad y sexo, así como a CF preoperatorio y necesidad de revascularización miocárdica concomitante. Como quiera que sea, esta diferencia probablemente obedezca a que en nuestra institución, al igual que en todos los grandes centros, una vez introducida la reparación mitral, de acuerdo a los principios de Carpentier y con control ecocardiográfico transesofágico intraoperatorio en todos los casos, ésta rápidamente se impuso por sobre el reemplazo, como tratamiento quirúrgico de la insuficiencia mitral ${ }^{7-10}$. Por lo mismo, en la actualidad no es concebible un estudio prospectivo y aleatorio para comparar ambos procedimientos.

Técnica quirúrgica. En esta experiencia se respetaron estrictamente los principios generales y específicos establecidos por Carpentier para la 
reparación de la válvula mitral insuficiente ${ }^{1-3}$. Especialmente, se utilizó un anillo circunferencial de Carpentier-Edwards, en la gran mayoría de los casos con el modelo Classic, que fue con el que Carpentier y todos los grupos importantes del mundo iniciaron y desarrollaron su experiencia en cirugía reconstructora de la insuficiencia mitral ${ }^{1-}$ 3,5,6,15-18. Esta anuloplastia pretende remodelar el anillo mitral, devolviéndole su forma original de «iñón», a la vez que aumentar la coaptación de los velos mitrales, disminuyendo el diámetro anteroposterior del plano valvular mitral, y reforzar las suturas anulares, para prevenir redilataciones tardías del anillo mitral ${ }^{1-3,16}$.

Mortalidad. Si bien todas las grandes series de la literatura muestran menor mortalidad operatoria para la reparación mitral, en nuestra experiencia esto no alcanzó significación estadística ${ }^{4-6,16-20}$. Sin embargo, la mortalidad global, incluyendo la operatoria, y en especial la mortalidad alejada de causa cardiovascular, fue significativamente menor para la reparación que para el reemplazo, en todo el período del seguimiento.

Reoperación. Nuevamente, en esta expeniencia no hubo diferencia significativa entre ambos procedimientos en la necesidad de reintervención mitral, si bien 2 pacientes con reparación y ninguno con reemplazo, requirieron de reintervención. Esto se contradice con las grandes series de la literatura en las que los pacientes con reparación mitral tendrían una menor necesidad de reintervención ${ }^{4-6,16-20}$. Lo antenor puede ser explicado por lo limitado de nuestra serie y en especial por la elevada mortalidad global de los pacientes con reemplazo mitral, puesto que al fallecer un paciente pierde el riesgo de reoperación.

Rehospitalización. La alta incidencia de rehospitalización por cualquier causa en ambos grupos de pacientes probablemente sea el reflejo de la avanzada edad de la mayoría de éstos, estando expuestos, por lo tanto, al desarrollo de variadas patologías, agudas y crónicas. Sin embargo, la acentuada diferencia en cuanto a rehospitalización por causas cardiovasculares entre ambos grupos debiera necesariamente estar relacionada al procedimiento quirúrgico, en especial a la mejor preservación de la función del ventrículo, y probablemente también de la aurícula izquierda, y a la menor necesidad de tratamiento anticoagulante de la reparación mitral ${ }^{4,17-20}$.

Eventos cardiovasculares. La combinación de muerte cardíaca, necesidad de rehospitalización cardiovascular y reoperación mitral fue marcadamente diferente para ambos procedimientos, siendo menor para la reparación mitral, durante todo el período de seguimiento. Nuevamente, esto debiera estar relacionado con la mantención de la función ventricular izquierda y la menor necesidad de tratamiento anticoagulante de la reparación mitrall $^{4,17,19}$. Sin embargo, no hubo diferencias en cuanto al estado sintomático de los pacientes, ya que al término del seguimiento todos los sobrevivientes, independientemente del procedimiento mitral, habían mejorado su $\mathrm{CF}$, encontrándose en ambos grupos todos ellos en CF I-II.

Limitaciones del estudio. Este es un estudio observacional descriptivo de 2 grupos de pacientes consecutivos operados en un período de 13 años y como tal tiene las limitaciones propias de las investigaciones retrospectivas, no aleatorias ni doble ciego. Más aún, los períodos de seguimiento son diferentes, puesto que la mayoría de los pacientes con reemplazo mitral fueron operados en los primeros años. Además, no incluye parámetros ecocardiográficos puesto que no fue posible obtenerlos en forma confiable para todos los pacientes, para todo el período en estudio. Sin embargo, a pesar de esto y a las diferencias numéricas de ambos grupos, los resultados clínicos son acentuados, lo que le debe dar validez a éstos. Además, lo anterior representa una situación real de cómo evolucionó el tratamiento de la insuficiencia mitral degenerativa en un servicio de cirugía cardiovascular importante del país. Como ya señalamos, en la actualidad no es concebible un estudio aleatorio de ambos procedimientos y de aquí la importancia para nuestro medio de un estudio como este.

En conclusión: esta experiencia, a pesar de las limitaciones señaladas, es concordante con la literatura internacional en cuanto a las ventajas de la reparación en comparación al reemplazo mitral, como tratamiento quirúrgico de la insuficiencia mitral degenerativa, lo que se tradujo en una menor mortalidad y morbilidad alejada de los pacientes con reparación mitral. 


\section{REFERENCIAS}

1. Carpentier A, Deloche A, Dauptain J, Soyer R, Blondeau P, Piwnica A et al. A new reconstructive operation for correction of mitral and tricuspid insufficiency. J Thorac Cardiovasc Surg 1971; 61: 1-13.

2. Carpentier A, Relland J, Deloche A, Fabiani J, D'Aluaines C, Blondeau P et al. Conservative management of the prolapsed mitral valve. Ann Thorac Surg 1978; 26: 294-302.

3. CarPentier A. Cardiac valve surgery - The «French correction». J Thorac Cardiovasc Surg 1983; 86: 323-37.

4. Отто CM. Evaluation and management of chronic mitral regurgitation. N Engl J Med 2001; 345: $740-$ 6.

5. Cosgrove D. Surgery for degenerative mitral valve disease. Semin Thorac Cardiovasc Surg 1989; 1: 183-93.

6. David TE, Armstrong S, Sub Z, Daniel L, Late results of mitral valve repair for mitral regurgitation due to degenerative disease. Ann Thorac Surg 1994; 56: 7-14.

7. Zalaquett R. Cirugía reparadora de la insuficiencia valvular mitral. Rev Chil Cir 1994; 46: 127-36.

8. Zalaquett R, Morán S, Irarrázaval MJ, Maturana G, Braun S, Chamorro G ET aL. Reconstrucción válvula mitral. Cirugía reparadora de la insuficiencia valvular mitral con evaluación ecocardiográfica transesofágica intraoperatoria. Rev Chil Cardiol 1996; 15: 78-84.

9. Zalaquett R, Chamorro S, Braun S, Garrido L, Howard M, Morán S ET AL. Resultados alejados de la cirugía reconstructora de la insuficiencia mitral. Rev Méd Chile 1999; 127: 1093-100.

10. Zalaquett R, Camplá C, Córdova S, Braun S, Chamorro G, Irarrázaval M et al. Resultados alejados de la cirugía reparadora de la insuficiencia mitral degenerativa. Rev Méd Chile 2003; 131: 1355-64.

11. Zalaquett R, Becker P, Irarrázaval MJ, Morán S, Maturana G, Navarro M et al. Cardioplejia retró- grada por canulación transauricular derecha del seno coronario. Rev Chil Cardiol 1993; 12: 68-71.

11. Zalaquett R, Morán S, Irarrázaval MJ, Maturana G, Navarro M, Braun S ET al. Acceso auricular izquierdo transeptal y superior combinado. Rev Chil Cir 1993; 45: 225-8.

12. Edmunds LH, Ciark RE, Cohn LH, Miler DC, Weisel $\mathrm{RD}$. Guidelines for reporting morbidity and mortality after cardiac valvular operations. J Thorac Cardiovasc Surg 1988; 96: 351-3.

13. Zalaquett R, Garrido L, Morán S, Irarrázaval MJ, Becker P, Maturana G et al. Cirugía de la insuficiencia mitral isquémica. Rev Méd Chile 2002; 130: 9-16.

14. Carpentier AF, Lessana A, Reland JY, Bem E, Mihaileanu S, BerReBi AJ et al. The «Physio-Ring» An advanced concept in mitral valve annuloplasty. Ann Thorac Surg 1995; 60: 1177-85.

15. Tribouiloy CM, Enríquez-Sarano M, SchafF HV, Orszulak TA, Bailey KR, Tajik J et al. Impact of preoperative symptoms on survival after surgical correction of organic mitral regurgitation. Rationale for optimizing surgical indications. Circulation 1999; 99: 400-5.

16. Galoway AC, Colvin SB, Baumann G, Espósito R, VOHRA R, HARTY S ET AL. Long-term results of mitral valve reconstruction with Carpentier techniques in 148 patients with mitral insufficiency. Circulation 1988; 78 (suppl):I 97-105.

17. Cosgrove DM, Chávez AM, Lytle BW, GiLl CC, Stewart RW, TAYLOR PC ET aL. Result of mitral valve reconstruction. Circulation 1986; 74 (suppl):I 827.

18. Galloway AC, Colvin SB, Baumann FG, Grossi EA, RiBakove GH, HaRTy S et al. A comparison of mitral valve reconstruction with mitral valve replacement: intermediate-term results. Ann Thorac Surg 1989; 47: 655-62.

19. Grossi EA, GaLoway aC, Miuer JS, Ribakove GH, CULFORD AT, Espósito R ET AL. Valve repair versus replacement for mitral insufficiency: when is a mechanical valve still indicated? J Thorac Cardiovasc Surg 1998; 115: 389-96.

\section{Agradecimientos}

Los autores agradecen a las bioestadísticas señoritas Paola Viviani y Alessandra Gederlini y a su secretaria señorita Verónica Delgado, por su asistencia estadística y las múltiples correcciones del manuscrito. 